\title{
CAPACIDADES CIENTÍFICAS EN EL TRASTORNO DEL ESPECTRO AUTISTA EN MÉXICO: REFLEXIONES DESDE EL VÍNCULO ENTRE CIENCIA, TECNOLOGÍA E INNOVACIÓN Y POLÍTICAS SOCIALES
}

\section{Scientific capacities in Autism Spectrum Disorder in Mexico. A contribution from science and technology policies}

\author{
Mónica Anzaldo Montoya \\ Investigadora de la Cátedra Conacyt en el Programa de Estudios Antropológicos de El Colegio de San Luis Potosí, \\ México. https://orcid.org/0000-0002-0164-2772
}

\section{Laura Patricia Cruz Ruiz}

Docente en el Instituto para Nińos Superdotados Dr. Oschmann GmbH (Bochum) \& inti -Sociedad para la

Integración Escolar (Dortmund), Alemania. https://orcid.org/0000-0001-5770-5974

Recibido: 22/1/2019 • Aprobado: 12/2/2019

Cómo citar: Anzaldo Montoya, M., \& Cruz Ruiz, L. P. (2019). Capacidades científicas en el Trastorno del Espectro Autista en México: reflexiones desde el vínculo entre ciencia, tecnología e innovación y políticas sociales. Ciencia y Sociedad, 44(1), 13-26. https://doi.org/10.22206/cys.2019.v44i1.pp13-26

\section{Resumen}

En el campo de los estudios sobre ciencia, tecnología y sociedad prevalece la discusión acerca de cómo direccionar las capacidades científicas a las necesidades sociales. Una problemática escasamente considerada como ámbito de orientación del conocimiento, son las necesidades de las personas con autismo y sus familias. El autismo afecta a 1 de cada 160 niños a nivel mundial y es la condición de mayor prevalencia en el ámbito psiquiátrico. México carece de políticas de CTI que articulen las necesidades de esta población. Este trabajo aporta un primer análisis de las capacidades científicas y la financiación pública dedicado al tema por el período de 1985-2018. A partir de herramientas bibliométricas y datos empíricos, se encontró un campo de investigación rezagado en producción académica, pero se identifica una comunidad creciente y vinculada con actores sociales y empresas privadas. Finalmente, se ofrecen recomendaciones para conformar una agenda de investigación.

Palabras clave: capacidades científicas en autismo; políticas de ciencia y tecnología; autismo en México; Trastorno del Espectro Autista.

\begin{abstract}
In the field of studies in science, technology and society, the discussion about how to guide scientific capacities to address social demands prevails. The problem is that it is hardly considered as an area of orientation in scientific knowledge. Mexico citizens are depleted overall with support and services to care for the needs of the people living with autism and their family. Worldwide, autism affects 1 of each 160 children and currently is the most prevalent condition in the Psychiatric field. Mexico lacks epidemiologist data and CTI policies that articulate the needs of this population. The objective of this paper is to carry out a first analysis of scientific capacities and public funding this topic from 1985 to 2018. Bibliometric tools and empirical data, institutions, topics and research project will be analyzed. Finally, recommendations will be suggested to propose a research agenda.
\end{abstract}

Keywords: scientific capacities in autism; science and technology policy; autism in México; Autism Spectrum Disorder. 


\section{Introducción}

En el campo de los estudios sobre ciencia, tecnología y sociedad (CTS) en América Latina prevalece la discusión acerca de cómo orientar las agendas de investigación e innovación a la atención de necesidades concretas de la sociedad, ya que hasta el momento no se ha logrado que las políticas de ciencia, tecnología e innovación (CTI) contribuyan de forma más directa a la solución de problemas que afectan a los más excluidos (Lemarchand, 2010; Albornoz, 2013; Casas, 2015). La literatura sobre el tema seńala que una de las principales limitantes para que los sistemas de investigación atiendan de manera prioritaria y sistémica las necesidades sociales es la fuerte orientación que han tenido las políticas de CTI hacia las demandas del mercado. Lo anterior se ampara bajo la premisa de que las actividades de $\mathrm{I}+\mathrm{D}$ en las instituciones públicas son un insumo que contribuye a la capacidad de innovación de las empresas, tal como lo han mostrado los aportes de la economía de la innovación (Nelson, 1959; Nelson, 1993; Johnson \& Lundvall, 1994).

Diversos estudios han problematizado esta cuestión y dentro de sus conclusiones señalan que, si bien las políticas de CTI se establecen en términos de atender las necesidades de salud, medioambiente o pobreza, en la práctica los programas de financiamiento a las actividades de investigación continúan dando mayores incentivos a la explotación comercial de los conocimientos, lo cual deja poco margen de apoyo para proyectos de investigación que no entran en esa formulación (Thomas, Fressoli \& Santos, 2012; Casas et al., 2013; Dutrénit \& Sutz, 2013).

Existe evidencia de que la generación de conocimiento es una estrategia de intervención legítima y fundamental para visibilizar los problemas sociales y modificar la realidad social (Bernal, 1979; Ziman, 2003; Kreimer \& Zabala, 2006). Los actores académicos pueden contribuir en la visibilización de los problemas sociales, acercarlos a la agenda pública para que puedan debatirse y, eventualmente, diseñar una política pública que marque el camino hacia su atención.

En este contexto, una problemática escasamente considerada como ámbito de orientación del conocimiento científico son las necesidades de las personas con autismo y sus familias. A pesar de que estudios recientes señalan un incremento mundial en su prevalencia, presentando un Trastorno del Espectro Autista (TEA) 1 de cada 160 nińos (Fombonne et al., 2016; OMS, 2017), y de que esta condición se ha convertido en uno de los padecimientos del desarrollo neurocientífico más frecuente en el ámbito psiquiátrico actual (Nicolini, 2018, p. 105), lo cierto es que las necesidades de las personas con autismo no ha sido un tema prioritario en las agendas científicas, al menos en lo que a América Latina se refiere.

En el caso de México, se carece de estudios epidemiológicos nacionales que informen sobre la prevalencia del autismo; sin embargo, un estudio reciente realizado en la ciudad de León, Guanajuato, muestra que cerca de un $1 \%$ de la población infantil de esa ciudad nace con TEA (Fombonne et al, 2016, p. 1670). Asimismo, la necesidad de atención de niños con autismo en escuelas públicas de educación especial ha aumentado en una magnitud de 1,595 , en 2005 , a 14,723 , en 2017, lo que equivale a un .43\% de la matrícula de educación básica de 2017 (sEp, 2017). Por su parte, datos de la Clínica Mexicana de Autismo (CLIMA) analizados para esta investigación señalan un promedio de 30 casos nuevos de autismo al mes, solamente en una de sus sedes.

Dada esta situación, se sostiene que en México las necesidades de las personas con autismo se encuentran en una condición de invisibilidad social y su atención está muy alejada de la agenda de las políticas de CTI. Este trabajo argumenta que el nivel de producción científica sobre un problema social tiene una alta incidencia en su colocación como 
problema público; por tanto, en la medida en que un problema social es poco estudiado existen menos actores argumentando y realizando esfuerzos para integrarlo a la política pública. Dicho lo anterior y ante la ausencia de estudios similares, el objetivo de esta investigación es realizar un primer análisis de las capacidades científicas sobre el Trastorno del Espectro Autista (TEA) en México, a partir de la producción académica que obra en acervos bibliográficos internacionales y del financiamiento público destinado a atender esta condición. Este artículo expone los primeros resultados de una investigación en curso que analiza los procesos de producción y apropiación de conocimiento sobre TEA en México.

\section{El Trastorno del Espectro Autista: reseńa de sus controversias}

El autismo, actualmente reconocido como Trastorno del Espectro Autista (TEA), comprende un grupo de afecciones del neurodesarrollo tipificadas por dificultades de diferentes grados en las capacidades de interacción social, comunicación y un conjunto restringido de intereses y comportamientos repetitivos (APA, 2013; OMS, 2017). Se trata de una condición que se manifiesta en la infancia y permanece en la persona a lo largo de toda su vida, es decir que sus necesidades se van modificando a lo largo del tiempo, lo que implica una mayor complejidad en su atención.

El autismo fue identificado a principios del siglo pasado por la psiquiatra soviética Grunya E. Sukhareva, en 1926, (Manouilenko \& Bejerot, 2015) y, más tarde, por el psiquiatra austríaco Leo Kanner. En cuanto a su etiología, el autismo fue considerado por muchos ańos una enfermedad mental relacionada con la esquizofrenia; esto hasta la década de los noventa, en la que el Manual de Diagnóstico Estadístico de Trastornos Mentales DSM-IV, por sus siglas en inglés- lo incluye dentro de los Trastornos Generalizados del Desarrollo, junto a otros como el Trastorno de Rett, el Trastorno desintegrativo infantil y el Síndrome de Asperger (APA, 1995, p. 13).

La clasificación del autismo como un trastorno mental ha estado caracterizado por la controversia. Mary Langan (2011) hace un recuento de cómo desde la década de los sesenta los padres con niños dentro del espectro han luchado por buscar explicaciones científicas a las causas del autismo y con esto han logrado que la condición de sus hijos sea considerada en la agenda de salud de algunos países. La autora demuestra el papel fundamental de los padres en defender las teorías psicogenéticas del autismo en contra de aquellas cargadas de prejuicios, como la teoría de la llamada "madre refrigerador" de Bruno Bettelheim, la cual atribuía el autismo a las madres "poco amorosas" que propiciaban poco contacto físico con sus hijos.

Por su parte, la literatura reciente considera al autismo un desorden neurológico de amplio espectro que puede estar asociado con factores genéticos y del medio ambiente (Oviedo et al., 2015). A estas hipótesis se suman nuevos estudios que lo asocian con la exposición a elementos tóxicos durante el embarazo, a los cultivos de organismos genéticamente modificados, como lo menciona Talk About Curing Autism (TACA), quien destaca la presencia de glifosato en cultivos como soya, maíz, canola, azúcar (TACA, "GMos and ASD", "s.f.") o a la inflamación sistémica del tracto gastrointestinal, que estaría afectando el cerebro, el sistema inmunitario y el metabolismo (Harrison et al., 2015). En síntesis, como campo de investigación, los estudios sobre autismo se encuentran en un momento de anomalías, en donde el paradigma científico dominante se ha movido del campo psiquiátrico y psicológico al genético, y de este al nutricional y al biomédico.

\section{Metodología}

El diseńo de este estudio es de corte cuantitativo, de alcance exploratorio y deductivo. Previo al 
diseño metodológico se realizaron entrevistas a profesionales con experiencia en el tema y a padres de personas con autismo. Se utilizaron principalmente dos estrategias metodológicas: un estudio bibliométrico y la conformación de un corpus de datos de fuentes oficiales solicitadas a las dependencias gubernamentales que pudieran tener a cargo proyectos de atención a la población con TEA. El estudio bibliométrico es una herramienta que comprende el tratamiento estadístico de los datos bibliográficos incluidos en las publicaciones artículos, libros, patentes, conferencias, entre otros-, cuya finalidad es aportar elementos para comprender el progreso general de la ciencia (Sancho, 2002). Además, es una herramienta útil para estudiar la dinámica social de diversos campos científicos (Collazo et al., 2010; Hernández, Kleiche, Russel, 2013; Anzaldo, Chauvet \& Maldonado, 2014). Cabe mencionar que la recolección de la información se efectuó de diciembre de 2017 a marzo de 2018.

La base de datos bibliométrica (BdB) se construyó a partir de tres fuentes: Web of Science (wos), scopus y LILACS, esta última especializada en la producción científica en salud de América Latina y el Caribe, que cubre 924 revistas. Las ecuaciones de búsqueda para cada fuente fueron las siguientes: Para SCOPUS, Title: "autism" or "autistic" or "aspergers syndrome", and ABS: "autism" or "autistic" or "aspergers syndrome", and AFFILCOUNTRY: Mexico, la cual generó 69 registros. Para WoS: "autism" or "aspergers syndrome" or "autistic", and theme: "autism" or "aspergers syndrome" or "autistic", and address: Mexico not New Mexico, que generó 64 registros. Finalmente, para LILACS: tw: "autismo" or "síndrome de asperger" or "autista", and instance: "regional", and db: "LILACs", generándose 898 registros, que se depuraron para dejar únicamente aquellos que tuvieran como dirección del autor una institución mexicana, por lo que quedaron 4 registros. La búsqueda de los términos se delimitó a los campos de título, palabras clave y resumen; se eliminaron los trabajos que no fueran específicos, con la intención de identificar de manera muy rigurosa los trabajos sobre autismo en México.

Con los resultados de las tres plataformas se conformó una вDB de $\mathrm{N}=90$ documentos: 60 artículos y 30 proceedings o memorias de congresos. En el análisis consideramos tanto los artículos como los proceedings, agrupándolos con el nombre genérico de documentos.

\section{Resultados}

\section{Estudio bibliométrico}

En la gráfica 1 se presenta un panorama histórico de la investigación sobre el TEA en cuanto a los artículos publicados en revistas indexadas, el cual consta de 90 trabajos en el período comprendido desde 1985 hasta abril de 2018. El primer artículo llevó por título Themes of interest. Autism. Biological aspects, publicado en la Revista Salud Mental. Los resultados muestran una escasa producción científica a lo largo del período, aunque con una línea de tendencia positiva y un ligero repunte en 2013 y 2016. Se contabilizan, en promedio, 2.7 artículos por año. Los datos de 2018 son parciales, por lo que habrá que esperar para ver si la producción recuperará la tendencia creciente.

En la gráfica 2 se muestran las principales instituciones que han participado en la producción científica sobre TEA en México y su correspondiente número de autores por institución. En total, se identificaron 116 instituciones: 55\% de ellas son internacionales y $45 \%$ son nacionales. El estudio bibliométrico muestra que la Universidad Autónoma de Baja California (UABC) aportó 24 publicaciones, es decir, el 27\% de la producción; seguida del Centro de Investigación Científica y Educación de Ensenada (CICESE), con 16 publicaciones (18\%); y, en tercera posición, está la Universidad Nacional Autónoma de México (UNAM), con 11 publicaciones (12\%). El Instituto 
Gráfica 1. Evolución de la producción científica sobre el Trastorno del Espectro Autista en México, 1985-2018

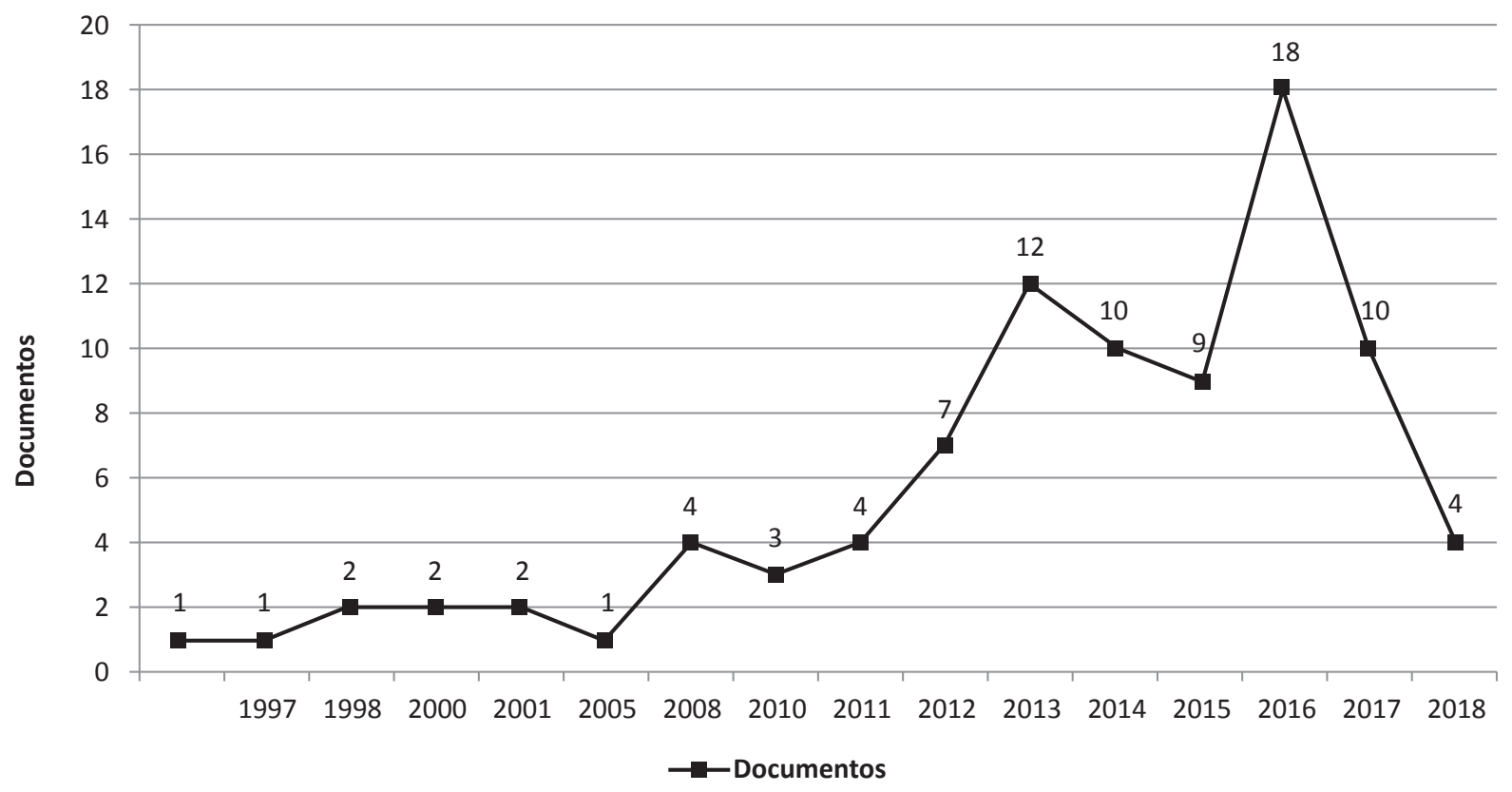

Fuente: elaboración propia a partir de sCOPus, wos y LILACS.

Nacional de Psiquiatría Ramón de la Fuente Muñiz y el Instituto Tecnológico de Tijuana aportaron un $8 \%$ de los trabajos.

En cuanto al número de autores, el resultado muestra una distribución distinta. El mayor número de investigadores tiene como adscripción la UNAM, seguido de la UABC, la Universidad Veracruzana y la Benemérita Universidad Autónoma de Puebla (BUAP). El total de autores participantes en el período es de 315 , lo cual equivale a un índice de 0.28 artículos por investigador. La creciente participación de la UABC y el CICESE se origina por una mayor producción de trabajos relacionados con ciencias de la computación y diseño de aplicaciones de apoyo terapéutico para personas con autismo.

El análisis bibliométrico también arrojó un panorama de la dinámica de producción científica sobre TEA; en esta se observa las vinculaciones que realizan los autores con asociaciones civiles, laboratorios de investigación corporativos y fundaciones, tanto de México como del extranjero. A nivel nacional, identificamos la colaboración con clínicas especializadas en problemas del desarrollo como CLIMA, Neuroingenia, el Instituto Domus, así como empresas como Softek y la Fundación Pasitos. Estas vinculaciones son para el desarrollo de aplicaciones que ayuden en la comunicación de las personas con autismo no verbales. En el ámbito internacional, encontramos que los trabajos publicados han recibido financiamiento del Banco Mundial, empresas como Microsoft, así como apoyos no económicos por parte de empresas como Nokia, Telefónica y Zinpro Co. La colaboración académica internacional está diversificada en 21 países; sin embargo, casi la mitad de los trabajos son en coautoría con instituciones de Estados Unidos (41\%), seguido del Reino Unido (10\%), Alemania y Canadá (4\%) e Italia (3\%). En contraste, la colaboración con países de América Latina es muy escasa; apenas dos 


\section{Gráfica 2. Principales instituciones en la producción científica sobre TEA en México, 1985-2018}

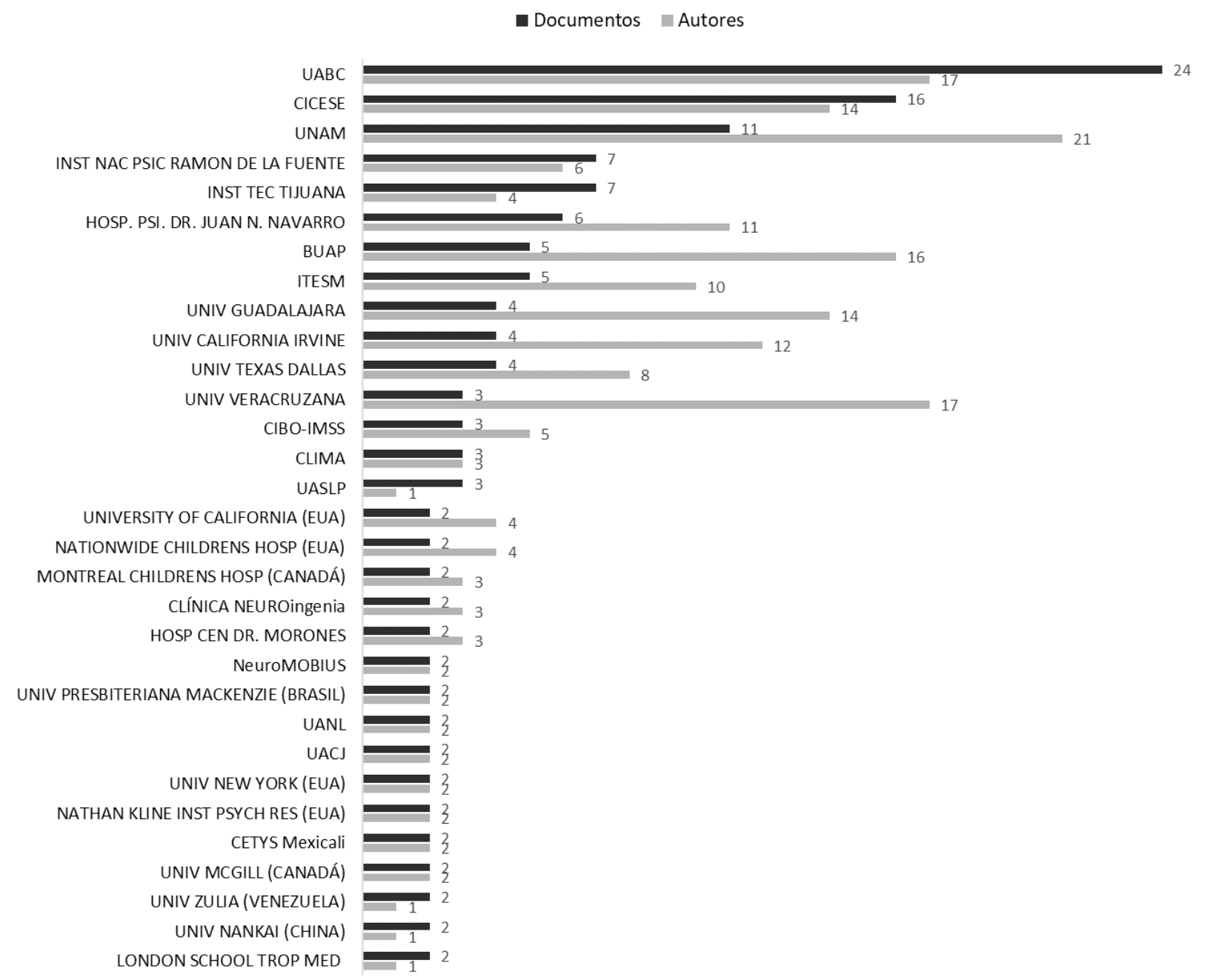

Fuente: elaboración propia a partir de scopus, wos y LILACS.

Nota: se graficaron solo las instituciones que contaran al menos con dos publicaciones.

publicaciones en coautoría con instituciones de Brasil, Chile y Venezuela, respectivamente.

A continuación, en la tabla 1 se presenta la distribución de los artículos según la revista o las memorias de congreso en la que fueron publicados. Únicamente se presentan las publicaciones con al menos dos registros. Para el caso de la revista, se señala su factor de impacto. El total de trabajos está disperso en 62 títulos, el $80 \%$ de los cuales es internacional, mientras que el $20 \%$ corresponde a editoriales nacionales. La revista con más registros es Salud Mental (6\%); le siguen las revistas Autism Research y el Journal of Autism and Developmental Disorders, junto con los trabajos presentados en Lecture Notes in Computer Science. Como puede apreciarse, las contribuciones se concentran en revistas especializadas en neurociencias y en ciencias computacionales, mientras que hay una ausencia de revistas de ciencias sociales, educación, salud pública, nutrición o ciencias de la conducta. En cuanto al factor de impacto, se observan algunas publicaciones en las revistas de mayor visibilidad en el campo. 
Tabla 1. Distribución de la producción científica sobre TEA por revista o conferencia internacional, 1985-2018

\begin{tabular}{lccc}
\hline \multicolumn{1}{c}{ Título de la revista o conferencia } & Factor de Impacto (2017) & Documentos & $\begin{array}{c}\text { \% de } \\
\text { N=90 }\end{array}$ \\
\hline Salud mental & Journal Citation Report & Docion & $6 \%$ \\
Autism research & 3.76 & 4 & $4 \%$ \\
Journal of autism and developmental disorders & 3.476 & 4 & $4 \%$ \\
Lecture notes in computer science & & 4 & $4 \%$ \\
Conference on human factors in computing systems & & 3 & $3 \%$ \\
leee pervasive computing & & 3 & $3 \%$ \\
Revista de neurología & 0.601 & 3 & $3 \%$ \\
Archivos de neurociencias & - & 2 & $2 \%$ \\
Eneurobiología & - & 2 & $2 \%$ \\
Gaceta médica de méxico & 0.342 & 2 & $2 \%$ \\
International journal of human-computer studies & 2.300 & 2 & $2 \%$ \\
Latin american conference on learning objects and technology, & & 2 & $2 \%$ \\
laclo & & 2 & $2 \%$ \\
Recent advances in human neurophysiology & & 2 & $2 \%$ \\
Revista chilena de neuropsicología & - & 2 & $2 \%$ \\
Revista mexicana de neurociencia & - & 2 & $2 \%$ \\
\hline Smart innovation, systems and technologies & & $2 \%$
\end{tabular}

Fuente: elaboración propia a partir de scopus, wos y LILACS.

\section{Análisis temático}

Para aproximarnos al conocimiento temático de la investigación sobre TEA se siguieron dos estrategias. Primero, un análisis de la distribución de los trabajos por grandes áreas disciplinarias; y, posteriormente, un análisis de frecuencia de palabras con los abstracts o resúmenes de las publicaciones, con la herramienta de análisis de contenido Voyant Tools. Consideramos adecuado el uso del resumen del artículo para tener una visión sintética de los temas que se investigan, ya que esta sección del artículo es una elaboración retórica que el autor utiliza para comunicar los resultados de su investigación y convencer a sus pares de la importancia de su contribución a la discusión científica.
En la tabla 2 se muestra la distribución de los documentos por grandes áreas disciplinarias. El mayor número de contribuciones se encuentra en Ciencias de la computación, con un $24 \%$, Neurociencias Neurología y Psicología, ambas con el $14 \%$, e Ingeniería, con un $10 \%$. Se observa la acumulación de trabajos en las disciplinas relacionadas con el desarrollo de aplicaciones tecnológicas, telecomunicaciones e ingeniería, mientras que en el área médica se observa la vocación por los temas relacionados con aspectos neurológicos y psiquiátricos del trastorno, mientras que los abordajes desde las ciencias de la conducta y las ciencias sociales están poco representados. 
Tabla 2. Distribución de la producción científica sobre TEA por grandes áreas disciplinarias

\begin{tabular}{lcc}
\hline Áreas de investigación & $\begin{array}{c}\text { Núm. } \\
\text { Documentos }\end{array}$ & $\%$ \\
\hline Ciencias de la com- & 31 & $24 \%$ \\
putación & 18 & $14 \%$ \\
Neurociencias neurología & 18 & $14 \%$ \\
Psicología & 13 & $10 \%$ \\
Ingeniería & 10 & $8 \%$ \\
Psiquiatría & 6 & $5 \%$ \\
Medicina & 6 & $5 \%$ \\
Otros & 5 & $4 \%$ \\
Telecomunicaciones & 4 & $3 \%$ \\
Ciencias de la conducta & 4 & $3 \%$ \\
Investigación educativa & 4 & $3 \%$ \\
Matemáticas & 4 & $3 \%$ \\
Ciencias sociales & 2 & $2 \%$ \\
Ciencias de la decisión & 2 & $2 \%$ \\
Pediatría & 2 & $2 \%$ \\
Fisiología & 2 & $2 \%$ \\
Ciencia, tecnología y otros & & \\
temas & $13 \%$ \\
Total & & $100 \%$ \\
\hline
\end{tabular}

Fuente: elaboración propia a partir de scopus, wos y LILACS.

En la gráfica 3 se presenta la nube de palabras contenidas en los resúmenes de los 90 trabajos que conforman la $\mathrm{BDb}$. Los términos más frecuentes se ubican al centro y tienen una tipografía de mayor tamaño. En este caso, el corpus de resúmenes contiene un total de 15,315 palabras y 3,161 palabras únicas, con una densidad de 0.206 , lo que indica un universo lexical reducido. Los términos que ocupan mayor centralidad son aquellos asociados a la caracterización sintomatológica del autismo: autism (autismo), children (niños), ASD (abreviatura de TEA en inglés), disorder (trastorno) y social (social). Pero, también se observa un conjunto de palabras con mayor frecuencia relativa como: "diagnóstico" y "tratamiento", así como aquellas relacionadas con el diseño de dispositivos tecnológicos, como son: user o users (usuario), model (modelo), technologies (tecnología), communication (comunicaciones), design (diseño). Al revisar con detenimiento los trabajos de este grupo se encontró que efectivamente se refieren al diseño de aplicaciones para tabletas o teléfonos inteligentes que ayudan a las personas con autismo, específicamente con limitación de habla, a comunicarse; mientras que otros son dispositivos que ayudan a controlar los comportamientos disruptivos de los alumnos en el aula.

Adicionalmente, con la finalidad de encontrar investigaciones sobre temas actuales en el ámbito del autismo, se procedió a buscar en el corpus palabras clave, tales como "adultos", "biomedicina", "alimentación", "tratamientos nutricionales", "dieta sin gluten y caseína”; de estas no se generó ningún resultado.

Gráfica 3. Nube de palabras a partir de los resúmenes de los trabajos de investigación sobre TEA

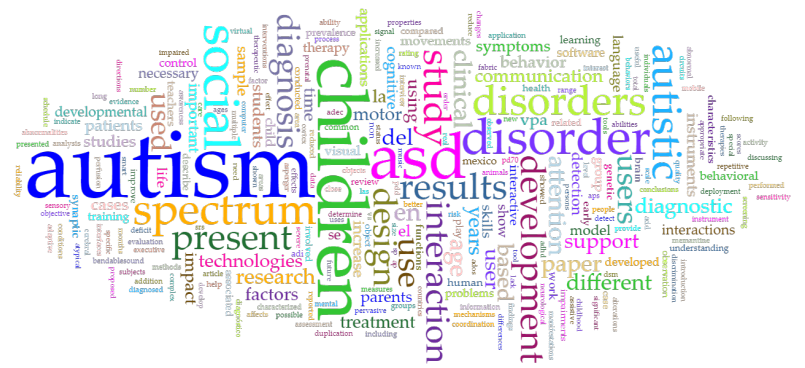

Visualización de 265 palabras de un total de 15,315. Las 20 palabras más frecuentes son: autism (202); children (128); asd (94); disorder (73); social (72); autistic (58); spectrum (55); disorders (54); study (47); interaction (43); development (41); design (39); diagnosis (37); use (37); users (34); attention (30); clinical (30); used (30); different (27); age (26).

Fuente: elaboración propia.

\section{Financiamiento a la investigación en TEA}

El análisis del financiamiento se realizó en dos aspectos; primero, se analiza cuáles instituciones 


\section{Gráfica 4. Instituciones que han financiado la investigación sobre TEA en México}

Datos a partir de las publicaciones de la base de datos bibliométrica:

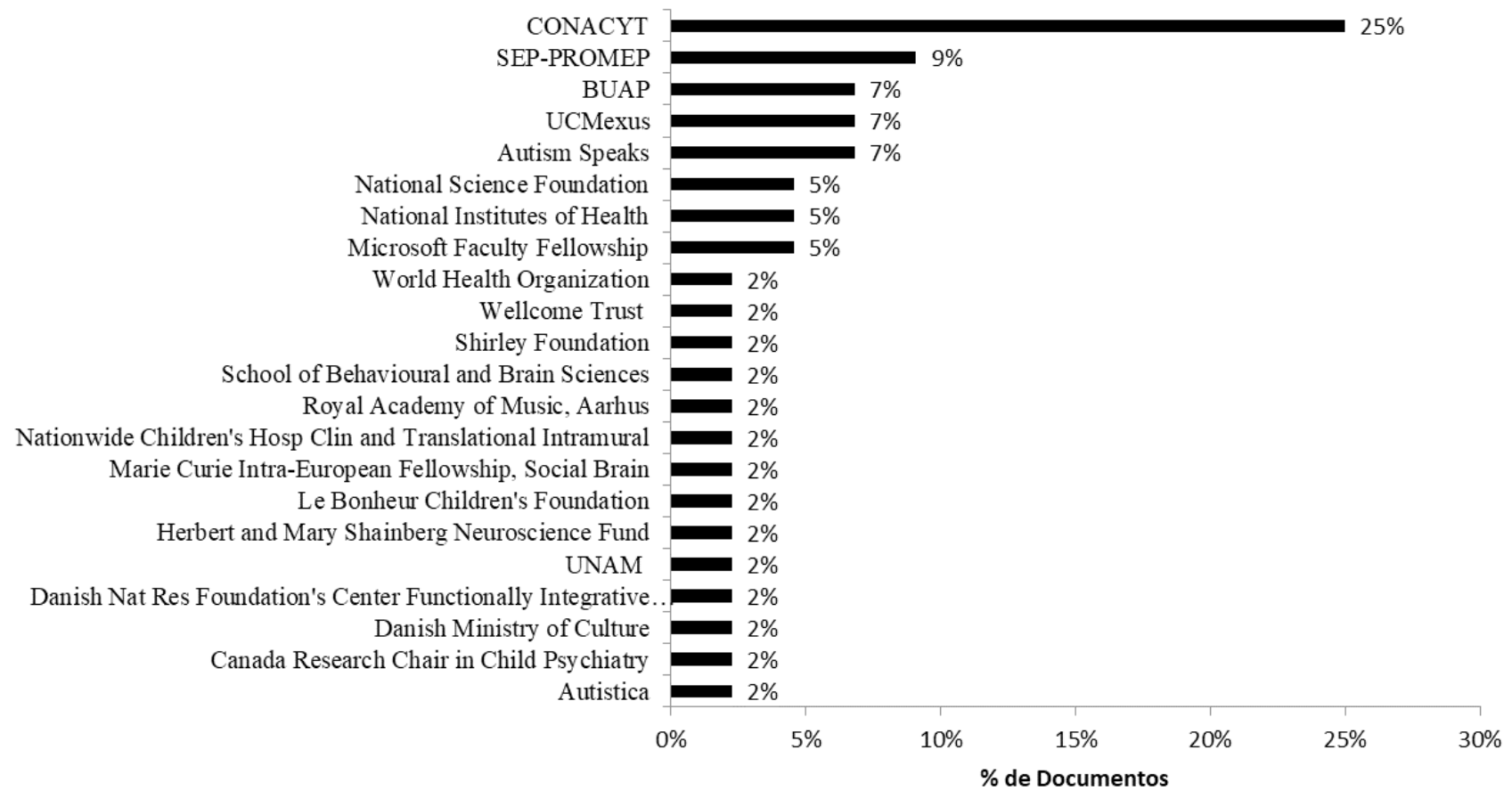

Fuente: elaboración propia a partir de scopus, wos y LILACS.

apoyaron los proyectos que dieron como resultado los artículos del estudio bibliométrico; en segundo lugar, cuál es el recurso otorgado por las entidades gubernamentales para proyectos de investigación. De los 90 trabajos que conforman la BDB, solamente 21 de ellos tuvieron financiamiento externo. De este universo se observa que el $43 \%$ corresponde a investigaciones que han sido apoyadas con recursos del Estado mexicano; el 57\% restante está compuesto por agencias y empresas de otros países, principalmente de Estados Unidos.

En la tabla 3 se presentan los dieciocho proyectos de investigación que han recibido financiamiento por parte del Estado. Se advierte que desde 2011 hasta 2017 el gobierno federal apoyó la investigación en autismo por un monto de $\$ 10,140,951$, lo que significa menos de un millón de pesos por proyecto de investigación en siete años. La principal institución financiadora es nuevamente el Conacyt (75\%), seguido por la Secretaría de Salud, a través del Fondo Sectorial de Investigación en Salud y Seguridad Social-Conacyt (18\%) y, en tercer lugar, la Secretaría de Salud en coinversión con la beneficencia pública $(6 \%)$.

Respecto de las temáticas de investigación, los resultados muestran como ejes de interés: el análisis etiológico del autismo en áreas disciplinares de la neurofisiología; el avance en la precisión del diagnóstico a partir de estudios metabólicos; el desarrollo de dispositivos tecnológicos para ayudas terapéuticas; $y$, en años recientes, la emergencia de un grupo de proyectos orientados al análisis y la evaluación de la infraestructura y las capacidades de atención en el diagnóstico temprano y la atención terapéutica, los cuales hemos denominado como de salud pública. 
Finalmente, se encontró que los proyectos de investigación tienen una intensa colaboración internacional con instituciones de Estados Unidos. Buena parte de los proyectos financiados por Conacyt están anclados a convocatorias bilaterales con ese país -UC-MEXUS, el programa ConTex con el Sistema de Universidades de Texas-, lo cual coincide con el comportamiento de coautoría mostrado en los hallazgos del estudio bibliométrico.

Tabla 3. Proyectos de investigación relacionados con trastorno del espectro autista financiados por instituciones gubernamentales, 2011-2018

\begin{tabular}{|c|c|c|c|c|c|}
\hline $\begin{array}{l}\text { Institución } \\
\text { financiadora }\end{array}$ & $\begin{array}{l}\text { Institución } \\
\text { beneficiada }\end{array}$ & Título del proyecto & $\begin{array}{l}\text { Temática que } \\
\text { atiende }\end{array}$ & $\begin{array}{l}\text { Monto (pesos } \\
\text { mexicanos) }\end{array}$ & Año \\
\hline \multirow{8}{*}{ CONACYT } & $\begin{array}{c}\text { Instituto Nacional de } \\
\text { Psiquiatría-Univ. Calif. } \\
\text { Riverside }\end{array}$ & $\begin{array}{l}\text { 1. UC-MEXUS: Alteraciones } \\
\text { relevantes en el Síndrome de } \\
\text { autismo debidas a la exposición } \\
\text { de éteres de polibromodifenilos } \\
\text { (PBDE) durante el desarrollo. } \\
\text { Participación del sistema } \\
\text { pacaergico. }\end{array}$ & $\begin{array}{c}\text { Etiología- } \\
\text { neurofisiología }\end{array}$ & 480 & 2011 \\
\hline & \multirow{2}{*}{$\begin{array}{l}\text { CICESE- Univ. Calif. } \\
\text { Irvine }\end{array}$} & $\begin{array}{l}\text { 2. UC-MEXUs: Enriqueciendo el } \\
\text { soporte visual interactivo con } \\
\text { modelado de video para niños } \\
\text { con autismo. }\end{array}$ & $\begin{array}{l}\text { Terapéutico/ } \\
\text { desarrollo } \\
\text { tecnológico }\end{array}$ & 480 & 2012 \\
\hline & & $\begin{array}{l}\text { 3. UC-MEXus: Mejorando } \\
\text { las terapias de música con } \\
\text { superficies interactivas para } \\
\text { apoyar la regulación sensomotriz } \\
\text { de niños con autismo. }\end{array}$ & $\begin{array}{l}\text { Terapéutico- } \\
\text { desarrollo } \\
\text { tecnológico }\end{array}$ & 480 & \multirow[b]{2}{*}{2015} \\
\hline & BUAP & $\begin{array}{l}\text { 4. Fondo Ciencia Básica: } \\
\text { efecto de la cerebrolisina en } \\
\text { los cambios en la morfología } \\
\text { cortical y la conducta en un } \\
\text { modelo animal de autismo } \\
\text { (exposición prenatal a ácido } \\
\text { valproíco en la rata). }\end{array}$ & $\begin{array}{c}\text { Etiología } \\
\text { Neurofisiología }\end{array}$ & 1,340 & \\
\hline & \multirow{2}{*}{ UNAM } & $\begin{array}{l}\text { 5. Fondo Ciencia Básica: } \\
\text { Plasticidad sináptica y } \\
\text { estructural en un modelo de } \\
\text { autismo. }\end{array}$ & $\begin{array}{c}\text { Etiología } \\
\text { Neurofisiología }\end{array}$ & 1,375 & \\
\hline & & $\begin{array}{l}\text { 6. Fondo Ciencia Básica: } \\
\text { Modelamiento del desarrollo } \\
\text { del lenguaje en el Síndrome } \\
\text { de Down y los trastornos del } \\
\text { espectro autista. }\end{array}$ & $\begin{array}{l}\text { Terapéutico } \\
\text { Modelamiento } \\
\text { Computacional }\end{array}$ & 504 & \multirow[t]{2}{*}{2016} \\
\hline & CICESE & $\begin{array}{l}\text { 7. Fronteras de la Ciencia: } \\
\text { Interfaces cerebrales en autismo. }\end{array}$ & $\begin{array}{l}\text { Desarrollo } \\
\text { Tecnológico }\end{array}$ & 948 & \\
\hline & $\begin{array}{c}\text { Universidad } \\
\text { Veracruzana-UT San } \\
\text { Antonio }\end{array}$ & $\begin{array}{l}\text { 8. CONTEX: The effects of natural } \\
\text { and social behavior on the } \\
\text { molecular and electrophysiological } \\
\text { properties of the Autistic } \\
\text { cerebellum. }\end{array}$ & $\begin{array}{c}\text { Etiología- } \\
\text { neurofisiología }\end{array}$ & 2,040 & 2017 \\
\hline
\end{tabular}




\begin{tabular}{|c|c|c|c|c|c|}
\hline $\begin{array}{l}\text { Institución } \\
\text { financiadora }\end{array}$ & $\begin{array}{l}\text { Institución } \\
\text { beneficiada }\end{array}$ & Título del proyecto & $\begin{array}{c}\text { Temática que } \\
\text { atiende }\end{array}$ & $\begin{array}{c}\text { Monto (pesos } \\
\text { mexicanos) }\end{array}$ & Año \\
\hline \multirow{3}{*}{$\begin{array}{l}\text { Fondo } \\
\text { Sectorial de } \\
\text { Investigación } \\
\text { en Salud y } \\
\text { Seguridad } \\
\text { Social ss/IMSs/ } \\
\text { ISSSTE-CONACYT }\end{array}$} & $\begin{array}{l}\text { Hospital Infantil } \\
\text { de México Federico } \\
\text { Gómez }\end{array}$ & $\begin{array}{l}\text { 9. Cambios en la conectividad } \\
\text { cerebral durante el estado de } \\
\text { reposo en el desarrollo infantil. }\end{array}$ & $\begin{array}{l}\text { Diagnóstico } \\
\text {-electrofisiología }\end{array}$ & 417 & 2012 \\
\hline & & $\begin{array}{l}\text { 10. Estudio del metaboloma } \\
\text { en orina y plaquetas en } \\
\text { enfermedades psiquiátricas. }\end{array}$ & $\begin{array}{l}\text { Diagnóstico- } \\
\text { metabólico }\end{array}$ & 692 & \multirow{2}{*}{2015} \\
\hline & $\begin{array}{l}\text { Instituto Nacional de } \\
\text { Medicina Genómica }\end{array}$ & $\begin{array}{l}\text { 11. Perfil inmunogenético } \\
\text { en pacientes diagnosticados } \\
\text { con espectro autista de origen } \\
\text { mexicano. }\end{array}$ & $\begin{array}{l}\text { Etiología- } \\
\text { metabólico }\end{array}$ & 740 & \\
\hline \multirow{3}{*}{$\begin{array}{l}\text { Secretaría de } \\
\text { Salud }\end{array}$} & $\begin{array}{l}\text { Inst. Nal. Psiquiatría } \\
\text { Ramón de la Fuente } \\
\text { Muñiz, Hosp. } \\
\text { Psiquiatría Infantil } \\
\text { Juan N. Navarro, } \\
\text { Hosp. Especialidades } \\
\text { Pediátricas Tuxtla } \\
\text { Gutiérrez, Chiapas }\end{array}$ & $\begin{array}{l}\text { 12. Trayectoria de atención y } \\
\text { demora diagnóstica en casos con } \\
\text { trastorno del espectro autista. }\end{array}$ & $\begin{array}{l}\text { Salud pública- } \\
\text { diagnóstico de } \\
\text { capacidades }\end{array}$ & $\mathrm{N} / \mathrm{D}$ & 2016 \\
\hline & Secretaría de Salud & $\begin{array}{l}\text { 13. Diagnóstico nacional de la } \\
\text { infraestructura disponible para } \\
\text { la atención a los trastornos del } \\
\text { espectro autista. }\end{array}$ & $\begin{array}{l}\text { Salud pública- } \\
\text { diagnóstico de } \\
\text { capacidades }\end{array}$ & $\mathrm{N} / \mathrm{D}$ & 2017 \\
\hline & $\begin{array}{l}\text { Secretaría de Salud, } \\
\text { Colima }\end{array}$ & $\begin{array}{l}\text { 14. Estudio sobre los recursos } \\
\text { y las estrategias utilizadas en } \\
\text { familias de poblaciones rurales } \\
\text { ante la presencia de un nińo con } \\
\text { alteraciones en el desarrollo. }\end{array}$ & $\begin{array}{l}\text { Salud pública- } \\
\text { diagnóstico de } \\
\text { capacidades }\end{array}$ & N/D & 2017 \\
\hline \multirow{4}{*}{$\begin{array}{l}\text { Secretaría } \\
\text { de Salud- } \\
\text { Beneficencia } \\
\text { pública }\end{array}$} & & $\begin{array}{l}\text { 15. Diagnóstico oportuno y } \\
\text { atención psicológica integral a } \\
\text { nińos y jóvenes con autismo y } \\
\text { otros trastornos del desarrollo } \\
\text { intelectual en Sinaloa. }\end{array}$ & $\begin{array}{l}\text { Salud pública- } \\
\text { terapéutico }\end{array}$ & 116 & 2012 \\
\hline & $\begin{array}{c}\text { Asociación Sinaloense } \\
\text { de Autismo }\end{array}$ & $\begin{array}{l}\text { 16. Atención terapéutica } \\
\text { integral a niños y jóvenes con } \\
\text { autismo y sus familias, para } \\
\text { lograr su plena integración a la } \\
\text { sociedad en Sinaloa. }\end{array}$ & $\begin{array}{l}\text { Salud pública- } \\
\text { terapéutico }\end{array}$ & 248 & 2014 \\
\hline & & $\begin{array}{l}\text { 17. Terapias de modificación de } \\
\text { conducta y lenguaje a niños y } \\
\text { jóvenes con autismo para lograr } \\
\text { su inclusión a la sociedad en } \\
\text { Sinaloa. }\end{array}$ & $\begin{array}{l}\text { Salud pública- } \\
\text { terapéutico }\end{array}$ & 153 & 2016 \\
\hline & $\begin{array}{l}\text { Asociación de ayuda a } \\
\text { niños con Trastornos } \\
\text { en el desarrollo }\end{array}$ & $\begin{array}{l}\text { 18. Atención temprana para } \\
\text { la rehabilitación de niños con } \\
\text { autismo en el Municipio de } \\
\text { Benito Juárez, Quintana Roo. }\end{array}$ & $\begin{array}{l}\text { Salud pública- } \\
\text { terapéutico }\end{array}$ & 129 & 2015 \\
\hline
\end{tabular}

Fuente: elaboración propia con información del Gobierno Federal. 


\section{Conclusiones}

Con relación al financiamiento público, los resultados muestran que los recursos asignados a este tema han sido muy limitados, con poco más de 10 millones de pesos mexicanos en siete años. El financiamiento proviene de instituciones alas queles corresponde esta función: el Conacyt y la Secretaría de Salud. Sin embargo, sorprende que instituciones como el Consejo Nacional para Prevenir la Discriminación, la Secretaría de Educación Pública o la Secretaría de Desarrollo Social no hayan diseñado un programa de investigación, pues la discriminación y la exclusión son de los principales retos que afrontan las personas con autismo en diferentes espacios - escuela, trabajo, etcétera-. Estos resultados confirman los señalamientos expuestos al inicio de este texto, en los que se sostiene que para que el conocimiento científico contribuya de mejor manera a la atención de las necesidades sociales es necesaria la transversalidad de la política de ciencia, tecnología e innovación con las políticas sociales.

Uno de los hallazgos más interesantes es que una parte importante de la producción científica y de los proyectos de investigación se relaciona con el diseño de desarrollos tecnológicos, básicamente para aplicaciones de apoyo terapéutico. Estas capacidades de investigación se encuentran en los estados del norte de la república y son impulsados por el CICESE, la UABC y el Instituto Tecnológico de Tijuana. Los resultados llaman la atención porque se podría esperar que el cúmulo de capacidades estuviera en las instituciones a cargo de la investigación en salud, aunque este comportamiento es reciente y acotado a la metodología utilizada.

Por otra parte, los datos empíricos recabados proporcionan elementos para la identificación de interesantes vinculaciones entre académicos, tecnólogos y las clínicas especializadas en autismo, lo cual es un avance en la atención de las necesidades de las personas con autismo, problemática que se planteó al inicio de este artículo.

Finalmente, consideramos que la condición del autismo en México requiere visibilizarse y atenderse como un asunto de salud pública, es decir, que integre conocimientos en el plano sociodemográfico, económico, de atención a la diversidad y de derechos humanos. Sostenemos que el conocimiento científico tiene que contribuir mucho en la mejora del bienestar de las personas que viven con esta condición. Una propuesta es la creación de una agenda nacional de investigación construida entre las personas con autismo y sus familias, los académicos, los médicos, las escuelas especiales, el sector educativo y el sector productivo. Dicha agenda deberá incluir una hoja de ruta a fin de que los resultados de investigación se traduzcan en políticas públicas en, al menos, las siguientes áreas: salud integral, desarrollo de habilidades e independencia y alimentación. Entre los temas urgentes que pueden enfrentarse con las capacidades actuales son: mejorar la orientación pediátrica para el diagnóstico temprano, la realización de un censo nacional de personas con autismo, realizar estudios epidemiológicos y abrir líneas de investigación transdisciplinaria.

\section{Referencias}

Albornoz, M. (2013). Innovación, equidad y desarrollo latinoamericano, Isegoría, (48), 111-126.

American Psychiatric Association [APA]. (1995). Manual diagnóstico y estadístico de los trastornos mentales. Barcelona: MASSON, S. A. Recuperado de: https://psicovalero.files.wordpress. com/2014/06/manual-diagnc3b3stico-y-estadc3adstico-de-los-trastornos-mentales-dsm-iv.pdf

American Psychiatric Association [APA]. (2013). Diagnostic and Statistical Manual of Mental Disorders: DSM-V. Arlington, VA: APA.

Anzaldo, M., Chauvet, M. \& Maldonado, L. (2014). Fondos públicos para la investigación en 
nanotecnologías en México y el cambio de paradigma de la política de ciencia, tecnología e innovación. Interciencia, 39(1), 8-15.

Bernal, J. D. (1979). Introducción. La ciencia en la historia. Ciudad de México: UNAM/Editorial Nueva Imagen. (Obra original publicada en 1954).

Casas, R., De Fuentes, C., Torres, A. \& Vera-Cruz, A. (2013). Estrategias y gobernanza del Sistema Nacional de Innovación Mexicano: retos para un desarrollo incluyente. En G. Dutrénit \& J. Sutz (ed.) (pp. 35-64). Sistemas de innovación para un desarrollo inclusivo. La experiencia latinoamericana. Ciudad de México: Foro Consultivo Científico y Tecnológico/LALICS.

Casas, R. (2015). Retos analíticos de las políticas de ciencia, tecnología e innovación para enfrentar la pobreza en América Latina. En R. Casas \& A. Mercado (Coords.). Mirada Iberoamericana a las politicas de CTI (pp. 259-296). Madrid: CLACso.

Collazo, F., Morales-Luna, M. E., Russel, J. M. \& Pérez-Angón, J. M. (2010). Enriching knowledge production patterns of Mexican physics in particles and fields, 85(3), 791-802.

Dutrénit, G. \& Sutz, J. (Ed.) (2013). Introducción. En G. Dutrénit \& J. Sutz (ed.). Sistemas de Innovación para un Desarrollo Inclusivo. La experiencia Latinoamericana (pp. 7-18). Ciudad de México: Foro Consultivo Científico y Tecnológico/ LALICS.

Fombonne E., Marcin C., Manero A. C., Bruno R, Díaz C, Villalobos M., Ramsay K. (2016). Prevalence of Autism Spectrum Disorders in Guanajuato, Mexico: The Leon Survey. Journal of Autism and Developmental Disorders, 46(5), 1669-1685.

Harrison, J., Maun, C. Schaefer, N. M., de Laosa, M. (2015). A review of gluten- and casein-free diets for treatment of autism: 2005-2015. Nutrition and Dietary Supplements, 7, 87-101.
Hernández, Y., Kleiche, M. \& Russel, J. M. (2013). Enfoques metodológicos para identificar y caracterizar la investigación mexicana en química en bases de datos bibliográficas. Investigación Bibliotecológica, 27(59), 35-66.

Kreimer, P. \& Zabala, J. P. (2006). ¿Qué conocimiento y para quién? Problemas sociales, producción y uso social de conocimientos científicos sobre la enfermedad de Chagas en Argentina, Redes, 12 (23), 49-78.

Johnson, B. \& Lundvall, B. (1994). Sistemas nacionales de innovación y aprendizaje institucional, Comercio exterior, 44(8), 695-704.

Langan, M. (2011). Parental voices and controversies in autism. Disability \& Society, 26(2), 193-205.

Lemarchand, G. A. (2010). Sistemas nacionales de ciencia, tecnología e innovación en América Latina y el Caribe, unesco Estudios y documentos de política científica en ALC Vol. 1, Montevideo: unesco.

Manouilenko I. \& Bejerot, S. (2015). S. Sukhareva - Prior to Asperger and Kanner. Nordic Journal of Psychiatry, 69, 479-482.

National Health Center for Health Statistics (NHCS) (2016). Estimated Prevalence of Children With Diagnosed Developmental Disabilities in the United States, 2014-2016. Recuperado de: https:// www.cdc.gov/nchs/data/databriefs/db291.pdf

Nelson, R. R. (1959). The simple economics of basic scientific research. Journal of political economy, 67(3), 297-306.

Nelson, R. R. (1993). National Innovation System: a Comparative Analysis, Oxford: University Press.

Nicolini, H. (2018). Autism, the current pathological trend in psychiatry, Salud Mental, 41(3), 105-107.

Organización Mundial de la Salud (oms). (2017). Trastornos del espectro autista. Recuperado de 
https://www.who.int/es/news-room/fact-sheets/ detail/autism-spectrum-disorders

Oviedo, N., Manuel-Apolinar, M., Dela Chesnayea, E. \& Araiza, Ch. (2015). Aspectos genéticos y neuroendocrinos en el trastorno del espectro autista, Boletín Médico del Hospital Infantil de México, 72, 5-14. DOI: 10.1016/j.bmhimx.2015.01.010

Sancho, R. (2002). Indicadores de los sistemas de ciencia, tecnología e innovación. Economía Industrial, 343, 97-109.

Secretaría de Educación Pública. (2017). Estadística e indicadores educativos; 2017. [consultado el 3 de mayo de 2018 ] Recuperado de: http://www.snie. sep.gob.mx/descargas/estadistica_e_indicadores/ estadistica_e_indicadores_educativos_15MEX.pdf

Thomas, H., Fressoli, M. \& Santos, G. (2012). Tecnología, Desarrollo y Democracia. Nueve estudios sobre dinámicas sociotécnicas de exclusión/inclusión social. Buenos Aires Ministerio de Ciencia, Tecnología e Innovación Productiva de la Nación.

Ziman, J. (2003). Ciencia y sociedad civil. Isegoría, (28), 5-17.

\section{Datos de filiación}

Dra. Mónica Anzaldo Montoya, investigadora Cátedra Conacyt en el Programa de Estudios Antropológicos de El Colegio de San Luis. Sus líneas de investigación son gobernanza de la ciencia, la tecnología y la innovación así como políticas científicas.

Correo electrónico: monica.anzaldo@colsan.edu.mx

Laura Patricia Cruz Ruiz, doctora en Educación por la Universidad Ruhr (Bochum), Docente en el Instituto para Niños Superdotados Dr. Oschmann GmbH (Bochum).

Correo electrónico:1pcruz@hotmail.de 